\title{
Experimental analysis of the fall mitigation motion caused by tripping based on the motion observation until shortly before ground contact
}

\author{
Yasuhiro AKIYAMA*, Kento MITSUOKA*, Shogo OKAMOTO* and Yoji YAMADA* \\ * Depertment of Mechanical Systems Engineering, Nagoya University \\ Furo-cho, Chikusa-ku, Nagoya, Aichi, 464-8603, Japan \\ E-mail: yasuhiro.akiyama@mae.nagoya-u.ac.jp
}

Received: 26 November 2018; Revised: 7 March 2019; Accepted: 11 April 2019

\begin{abstract}
This study focuses on observing and analyzing the occurrence of a fall for humans during normal gait, which perhaps results in severe injury. Previously, the insufficiency of a recovery step, which diminishes the forward rotation of the body after tripping, was suggested as a factor for the failure of fall avoidance motion. However, although it was identified that a slow and/or short recovery step resulted in the failure of fall prevention, the physical process of tripping and falling during the gait was not analyzed sufficiently. In this study, the subject's reaction motion and fall process against tripping became clearer because the fall motion, which included the phase closer to the ground contact, could be recorded for a longer time than that in most previous studies. Although the subject attempted to mitigate the forward angular momentum and the descent of body induced by tripping by holding on his recovery foot, it was not effective, especially when the recovery step length was short. Among such trials, the larger forward inclination of the body, the excessive forward movement of the center of mass from the support point, and the smaller ground reaction force of the recovery foot in the normal direction was observed. Because the short recovery step resulted in the limitation of the moment arm, which affected the reaction torque of the recovery foot, it became difficult to decelerate the body rotation and prevent the fall. Furthermore, it was also suggested that the forward lean of the body and the decrease of the ground reaction force increased the impact speed and effective mass, which affected the impact force. The observation of such an advanced fall phase also contributes to a more realistic simulation of the human reaction motion for a more precise estimation of the fall injuries.
\end{abstract}

Keywords : Trip and fall, Reaction motion, Severity estimation, Fall experiment, Unavoidable fall

\section{Introduction}

Injuries caused by fall are one of the major risks for the elderly. A recent study indicates that approximately 10 to $30 \%$ of the elderly have experienced a fall (Niino et al., 2000). Furthermore, approximately $10 \%$ of those who fell were seriously injured and had limitations in their daily activity (Luukinen et al., 2000). Even after they were physically cured, the fear of the fall probably prolonged their inactivity, which resulted in a decrease of their quality of life (Arfken et al., 1994). Moreover, falls are a major cause of injuries for workers in factories or construction sites (Tate, 1992; Lipscomb et al., 2006).

Unfortunately, although the fall mechanism is studied to prevent and mitigate injuries, there are limited opportunities to observe a natural fall with sufficient measurement parameters (Robinovitch et al., 2013). Thus, the knowledge obtained in fall experiments is useful to understand fall motion quantitatively. The results of previous fall experiments suggest the existence of two fall avoidance strategies against tripping: elevating and lowering (Eng et al., 1994). Tripped people sometimes overcome an obstacle by elevating a tripped leg too soon without moving the position of the stance foot. Then, the elevated leg is stepped forward. Otherwise, people step the tripped leg on-site instantaneously and subsequently step the other leg forward. The former is the elevating and the latter is the lowering strategy. As mentioned above, a long 
forward step, which is called the recovery step, commonly appears among these strategies. According to the observation of the reaction motion of the elderly after tripping by Pavol et al., a quicker and longer recovery step was essential to stop the forward rotation and descent of the body to avoid a fall (Pavol et al., 2001). Other groups have also suggested the importance of the recovery step (Grabiner et al., 1993; Smeesters et al., 2001a).

However, the fall motion during a critical or terminal phase cannot be obtained in an experiment because it is unsafe for the subject, which means that it has to be intercepted before contacting the ground (Pavol et al., 2001; Owings et al., 2001) or performed in a designated area (Smeesters et al., 2001b). Otherwise, the fall avoidance motion must be used, which does not require an actual fall (Pijnappels et al., 2005; Eng et al., 1994). Meanwhile, although motion simulation is used to analyze the fall motion (DeGoede et al., 2003a; Lo et al., 2008), implementing fall avoidance and human mitigation motion into this simulation is difficult because of the imperfect study of such motions. One reason for the difficulty of simulating the fall motion is that the fall motion recorded in the experiment is too short, which means the simulation should start from the early phase of the fall (Zhou et al., 2002). Although a longer duration of motion should be simulated to estimate the reaction motion of humans, it is difficult to anticipate it.

Thus, the observation and analysis of the successive motion after the failed fall avoidance motion, which is the motion when the recovery step cannot stop the fall, is essential to understand the mechanism of the fall process and injuries caused by the ground contact. The present study conducted a series of fall experiments to observe the reaction motion against tripping, which resulted in the fall, to further understand the fall motion.

\section{Methods}

The experiment was performed with the permission of the institutional review board of Nagoya University.

\subsection{Subject}

Subjects were recruited from the student population of Nagoya University. Screening of the applicants revealed no gait disorders. Thus, all the applicants participated in the experiment. Seven healthy male young adults with a mean age of 21.4 years old and standard deviation (SD) of 1.2 participated in the experiment. Their mean height and weight were $172.9 \mathrm{~cm}( \pm 3.2 \mathrm{SD})$ and $61.0 \mathrm{kgf}( \pm 7.6 \mathrm{SD})$, respectively. Their average body mass index was 20.4 and ranged from 16.7 to 23.6 .

\subsection{Apparatus}

Each experiment was conducted in an approximate $5 \times 8 \mathrm{~m}$ area, in which motion was recorded at $100 \mathrm{~Hz}$ using a three-dimensional motion capture system (MAC 3D System, Motion Analysis Corporation, US). A total of 37 markers, including cluster markers, were attached, as shown in Fig. 1. Subsequently, a pole was hung in front of the subject and moved at a constant speed, in between 1.3 to $1.4 \mathrm{~m} / \mathrm{s}$, using an electric motor to stabilize the subject's gait speed and prevent the subject from reducing the gait speed after the tripped trial, which was observed in the preliminary experiment. In addition, a metronome was used to maintain a constant gait rhythm. The rhythm of the metronome was tuned for each subject to be able to walk naturally.

The ground reaction force (GRF) was recorded at $100 \mathrm{~Hz}$ using four mobile six-axis force plates (M3D, Tech Gihan Co., Ltd., Japan) fixed under the toe and the heel of both feet. A bar was fixed in the lane from the side at a height of $25 \mathrm{~cm}$ to trip the subject. Some previous studies used a flat plate with a height of approximately $10 \mathrm{~mm}$ (Eng et al., 1994; Pijnappels et al., 2001; Wang et al., 2012). However, a bar, which also causes falls in the daily living environment, was selected to increase the probability of a fall in this study. The contact force on the bar was measured using two three-axis force sensors (USL08-H6-2KN-C, Tech Gihan Co., Ltd., Japan). The subjects wore goggles with the lower half covered, to hide the bar from sight and prevent the anticipation of tripping. Moreover, each subject's ankle was connected to a static object using a rope to control the length of the recovery step after tripping. Its tension force was measured by a load cell (RSCC-200 kg, Unipulse Corporation, Japan). Because the subject employed the "lowering" strategy in all preliminary trials, the rope was connected to the recovery leg, which was the opposite leg to the tripped side. The safety harness was hung using an air spring from the gondola, which moved along the lane with the pole using an electric motor. The safety harness did not interfere with the gait motion of the subject because it slacked in the standing posture. Another load cell fixed to the harness was used to measure the supporting force. Figure 2 shows the experimental setup. 


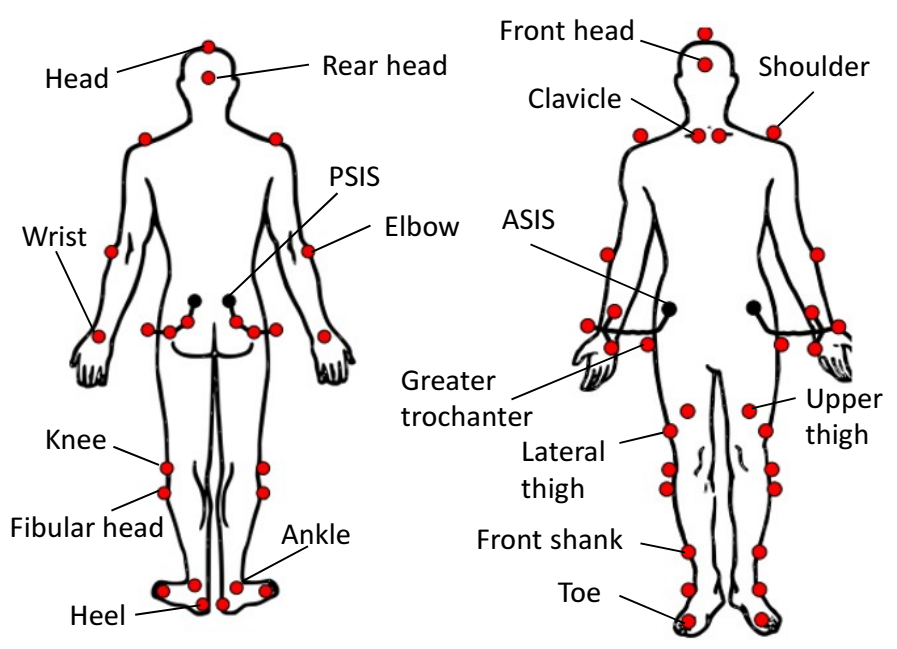

Fig. 1 Marker set Markers were attached to the top, rear, and front of the subject's head, shoulders, clavicle, elbows, wrists, anterior and posterior superior iliac spines (ASIS and PSIS), front and lateral thighs, knees, fibular heads, shanks, ankles, heels, and toes. Markers were attached on each feature point or at the rotation center of each joint. Markers on the right and left sides were attached symmetrically. Cluster markers, which was the rigid branch with three markers and estimated the position of the root using these markers, were used for superior iliac spines. A total of 37 markers were used.

\subsection{Protocol}

The experiment process was explained to each subject and each signed a consent form. Subjects wore well-fitted sportswear with reflective markers and shoes, in which force plates were installed. Safety gear and ropes were also attached. Then, the subject started to walk in the walking lane repeatedly.

The subjects were instructed to walk in a straight line to the hanging target placed at the extension of the walking lane. Before starting to walk, the subjects walked in place for five steps to adjust their gait timing to the metronome in each trial. Dummy trials, which did not include tripping, were randomly performed during tripping trials to prevent the subject's anticipation. The combinations of tripping conditions, which denoted the length of the limitation of the recovery step and the side of the tripped leg, were randomized. Thus, the position of obstacle and rope connection was switched for each condition between trials. In the tripped trials, two different restrictions regarding the recovery step, which had different limitations in terms of the step length, named short-step and long-step were used. A total of 35 trials included 23 non-tripped control cases, six short-step lengths, six long-step lengths were recorded for each subject. The number of tripped trials was the same for the right and left sides.

In this study, the position of the obstacle was adjusted to trip the subject in the middle to late swing phase, when the risk of injury becomes higher, as discussed previously (Mitsuoka et al., 2015; Dietz et al., 1986). However, the young adults seldom fell when tripped because they could perform sufficient recovery motion. According to a study on the failed recovery motion after tripping, the recovery step of fallers was shorter and slower than that of non-fallers (Pavol et al., 2001). Thus, in this study, a failed recovery step was artificially caused by limiting the step length of the recovery step using a rope, which connected the ankle of the subject to an object fixed at the rear part of the walking lane, to observe the fall motion constantly. The rope length was adjusted so that the recovery leg did not exceed the bar position in forward direction in the short-step case. The rope length was adjusted so that the recovery leg did not exceed the bar position in forward direction in the short-step case. In contrast, the rope length in the long-step case was extended by $20 \mathrm{~cm}$. Thus, the recovery step could become longer to resist forward falls more effectively. The rope length of each condition was adjusted beforehand to be the same as the position of the obstacle. The restriction force was monitored to identify whether the tripping condition was stable.

\subsection{Data Processing}

The recorded data were smoothed using a $6 \mathrm{~Hz}$ Butterworth filter. The trials, in which the marker was critically hidden, were not used in the analysis. The joint angles were calculated by fitting the motion of the markers to the human model of SIMM (Mulsculographics Inc., U.S.) using the least-squares method. The position of the center of mass (CoM) of the whole body was calculated using Zatsiorsky's method (De Leva, 1996). The trunk angle was determined as the angle of the line, which connected the center of the shoulder markers and the center of the hip markers, from the vertical 


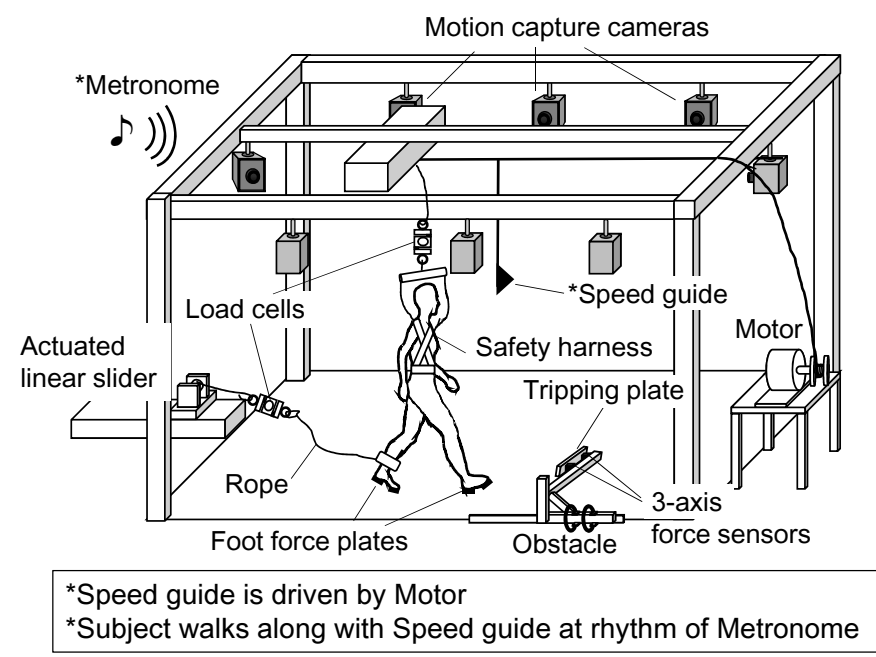

Fig. 2 Experimental setup A bar, which trips the subject, was fixed in the lane from either side. The subject's ankle was connected to a static object using a rope to control the length of the recovery step. The subject was supported by the safety harness when he fell.

direction (Wang et al., 2012). The GRF of each leg was calculated as the sum of both the toe and heel plates. The position of the center of pressure $(\mathrm{CoP})$ was calculated as the weighted center of GRF of each force plate. The position of each force plate in global coordinates was obtained from the motion capture. Then, CoM-CoP distance, which was the relative position of $\mathrm{CoM}$ from $\mathrm{CoP}$ in the traveling direction, was determined as the parameter of body balance and fall severity. The timing of the heel contacts and the toe offs was determined based on the normal direction of GRF with a $10 \mathrm{~N}$ threshold. The duration between successive heel contacts of the same leg was determined as the gait cycle (GC).

Fall trials were extracted from tripped trials and analyzed in this study. The trial, in which $50 \%$ of the body mass was supported by the harness, was determined as the fall trial. This threshold value was designed so that the subject could not avoid falling when the supporting force exceeded it. Furthermore, to avoid the effect of the harness tension on reaction motion, the motion before the force extracted at the harness exceeded $50 \mathrm{~N}$ were analyzed. The tripping timing was detected when the force exerted at the bar increased by $20 \mathrm{~N}$.

All trials herein were classified as the "lowering strategy," wherein the tripped leg was lowered and the other leg stepped forward too soon. This preference matched the previously reported relationship between the tripping phase and the fall avoidance strategy (Eng et al., 1994; Schillings et al., 2000). Four motion events were defined during the fall motion. The tripped timing (TRT) was defined as the timing of tripping. The subject lowered the tripped leg and touched the floor at the timing of the lowering step contact (LSC). The recovery step contact (RSC) was defined as the timing when the recovery leg touched the floor. Meanwhile, the timing when the support force of the harness exceeded the threshold owing to a subjects fall was defined as fall detected timing (FDT).

The GRF, step length, and CoM position were normalized based on the mass and height of each subject. The peak GRF was extracted from the lowering step and recovery step to evaluate the performance of fall mitigation. When evaluating the mean values of the gait parameters, we considered all trials uniformly. The difference of the motion parameter between the conditions was tested using the Mann-Whitney $U$-test.

\section{Results}

A total of 58 fall trials, which consisted of 29 short- and 29 long-step length cases, were analyzed. A typical fall motion is displayed in Fig. 3. All tripped trials were determined as the fall trial because the subject was finally fully supported by the safety harness. The trials with unintended trip timing, marker hiding, and other irregular motions were not processed. The average trip timing was $32.0 \pm 3.7 \% \mathrm{GC}$.

The gait parameters of normal walking trials were computed from all the subjects to validate the normal gait motion. The means and the SDs of the gait parameters were calculated as follows: walking speed, $1.34 \pm 0.07 \mathrm{~m} / \mathrm{s}$; cadence, $110.6 \pm 3.8 \mathrm{step} / \mathrm{min}$; step length, $73.1 \pm 6.6 \mathrm{~cm}$; and double support ratio, $20.0 \pm 2.6 \%$. Compared to a typical normal gait (Öberg et al., 1993), the subjects in the present experiment walked slightly slow with longer strides.

The tripping timing, peak impact force, and peak restricting force between the two recovery step length conditions 


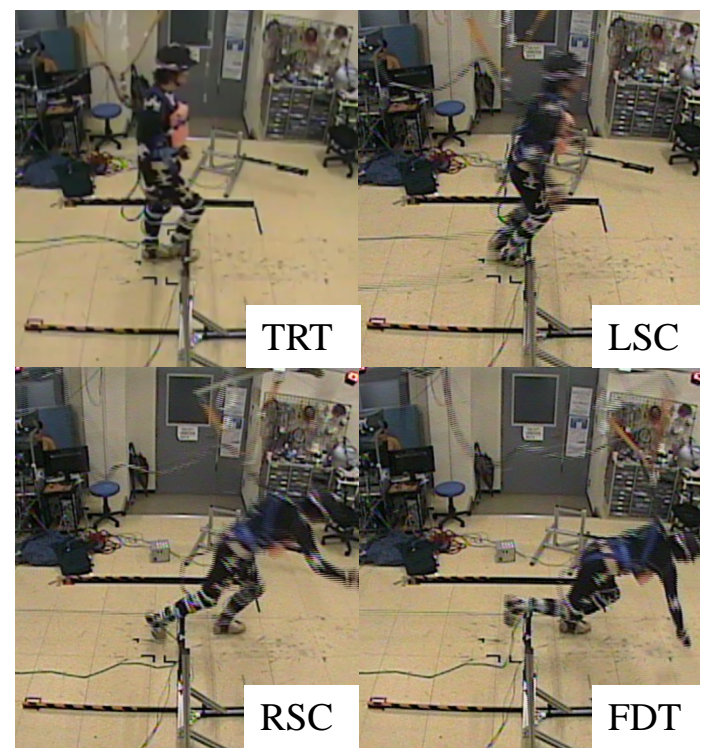

Fig. 3 Images of a typical falling motion observed in the experiment The posture of each timing, tripped timing, lowering step contact, recovery step contact, and fall detected timing is displayed. The right ankle of the subject tripped over the bar at TRT in this trial. Then, he lowered the tripped foot and it touched to the ground at LSC. Although the subject then moved his left leg forward, it was disturbed by the rope fixed to the left ankle. Thus, the step length of the recovery step was shortened at RSC. Finally, the subject was supported by the safety harness at FDT

did not significantly differ $(p>0.1)$. The recovery step lengths were $11.8 \pm 6.1 \%$ bh and $28.7 \pm 5.8 \%$ bh in the short- and long-step cases, respectively. In contrast, the timing of the recovery step after tripping did not significantly differ between the short $(0.53 \pm 0.08 \mathrm{~s})$ and long $(0.54 \pm 0.05 \mathrm{~s})$ steps.

The fall motions between the short- and long-step cases were compared in terms of the timing of the TRT, LSC, RSC, and FDT. Figures 4 to 6 show the boxplot of the representative fall motion parameters. Figure 7 summarizes the representative motion of the two conditions.

The fall progress is represented by the CoM height, trunk angle, and their velocities (Fig. 4 and Fig. 5(a) and (b)). A rapid decrease in the CoM height and a large CoM velocity at the timing of RSC and FDT expressed the descent of body as the fall progression. Furthermore, the CoM velocity in the traveling direction became lower at the RSC and FDT (Fig. 5(c)). The change of CoM velocity in the horizontal and vertical directions suggested that the gait speed transformed into a descent velocity when the fall progressed. At the same time, the increase in the trunk angle indicated the forward tilt, as shown in Fig. 7.

The CoM-CoP distance indicated the forward movement of the body from the support point, which occurred in the unbalanced posture before fall (Fig. 5(d)). When the CoM-CoP distance increased, the CoM lies excessively ahead of the support point because the supporting area could not expand owing to the insufficient recovery step, whereas the inertia moved the body forward. The peak normal GRF stood for the reaction force to stop the body descent that occurred during the fall progression (Fig. 6).

\section{Discussion}

The decrease of CoM height, increase of trunk angle, and transformation of CoM speed from horizontal direction to vertical direction were commonly observed among fall trials. Because these trends were those of the fall motion, which meant the subject could not compensate for the forward angular momentum imposed by tripping (Pijnappels et al., 2005), they commonly appeared regardless of the subject and rope length.

However, the difference of the recovery step length affected the rotation and descending speed of the fall as the fall progressed. Although the CoM height at the FDT did not significantly differ because the force threshold of fall detection was common, the difference of the CoM vertical velocity, which affected the fall injuries (DeGoede et al., 2002), became significantly higher in the short-step case (Fig. 5(b)). Instead, the CoM velocity in the traveling direction became lower at the RSC and FDT in the short-step case. These difference of CoM velocities suggested that the major direction of the motion of CoM differed between the short and long recovery steps because their magnitudes were almost the same. 


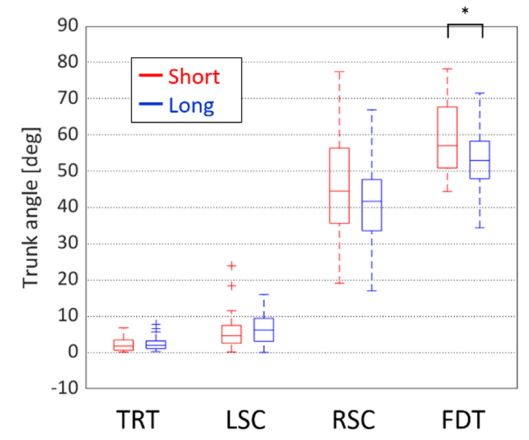

(a) Forward inclination angle of trunk

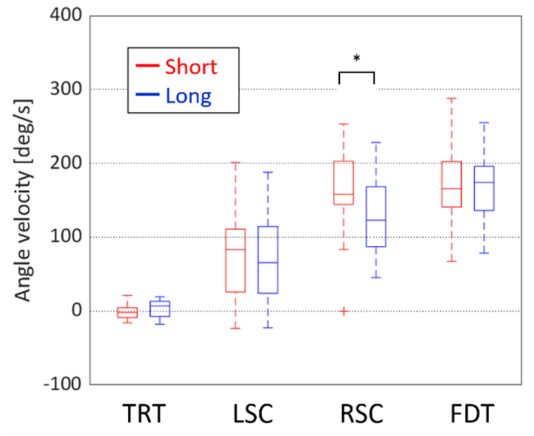

(b) Angle velocity of trunk inclination

Fig. 4 Body inclination and its velocity at the timing of fall events Box represents the first and third quartiles. Outliers, which are separated from the quartile more than 1.5 times the interquartile range, are marked as a cross. $*: p<0.1, * *: p<0.01$

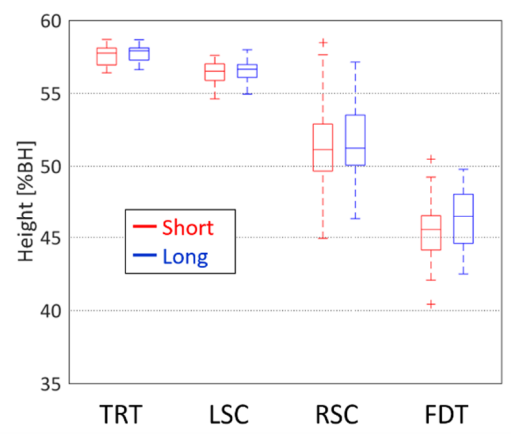

(a) CoM height

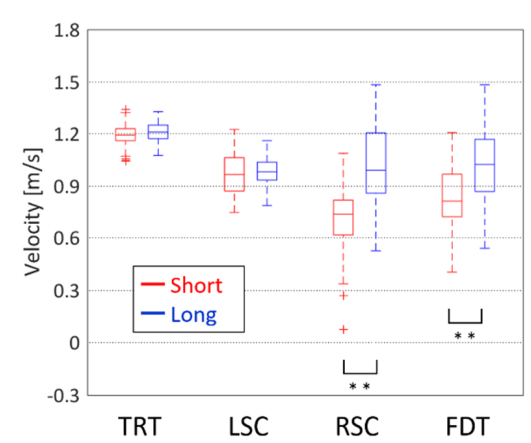

(c) CoM velocity in traveling direction

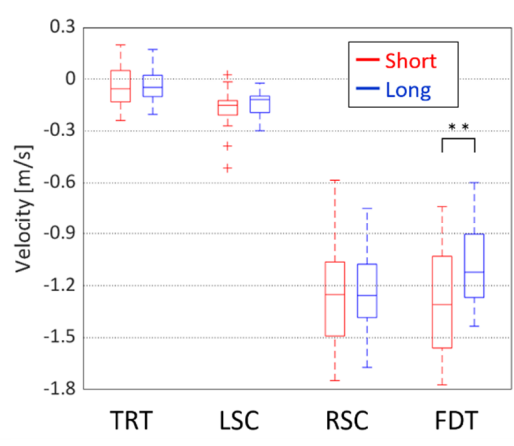

(b) CoM velocity in vertical direction

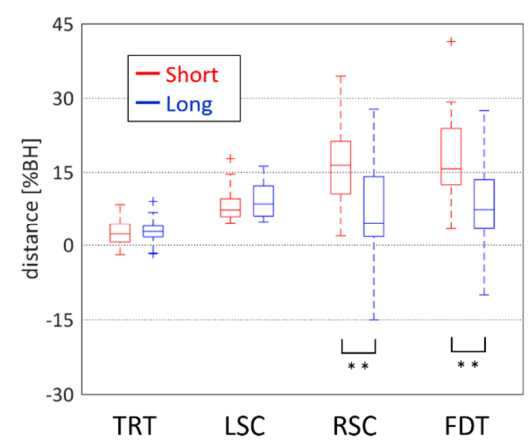

(d) Distance of CoM from CoP in traveling direction

Fig. 5 CoM position and motion at the timing of fall events Box represents the first and third quartiles. Outliers, which are separated from the quartile more than 1.5 times the interquartile range, are marked as a cross. $*: p<0.1, * *: p<0.01$

Furthermore, higher trunk inclination at FDT in the short-step case (Fig. 4) suggested that the forward angular momentum caused by tripping was not mitigated as effectively in the short-step case compared to the long-step case (Pavol et al., 2001).

The performance of mitigation motion could be considered from the CoM-CoP distance and the peak GRF. To decrease the impact force of ground contact, it was required to reduce the fall speed, which was represented by the vertical velocity of CoM. Thus, the large peak normal GRF, which helped to decelerate the descent of CoM, became essential. However, the large CoM-CoP distance made it difficult to exert GRF because of the loss of balance. Furthermore, when the CoM-CoP distance is large, the large GRF in the normal direction generates a moment toward forward rotation, which is another factor of a fall (Pavol et al., 2001; Pijnappels et al., 2005). Thus, pushing the ground to mitigate the descending 


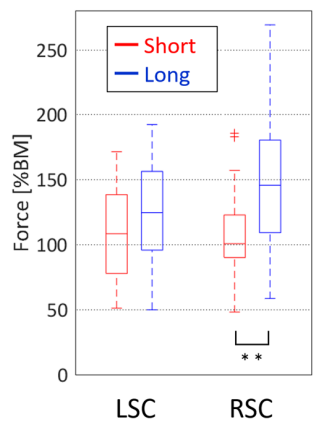

Fig. 6 Peak normal GRF at the timing of fall events Box represents the first and third quartiles. Outliers, which are separated from the quartile more than 1.5 times the interquartile range, are marked as a cross. ${ }^{*}: p<0.1$, $* *: p<0.01$

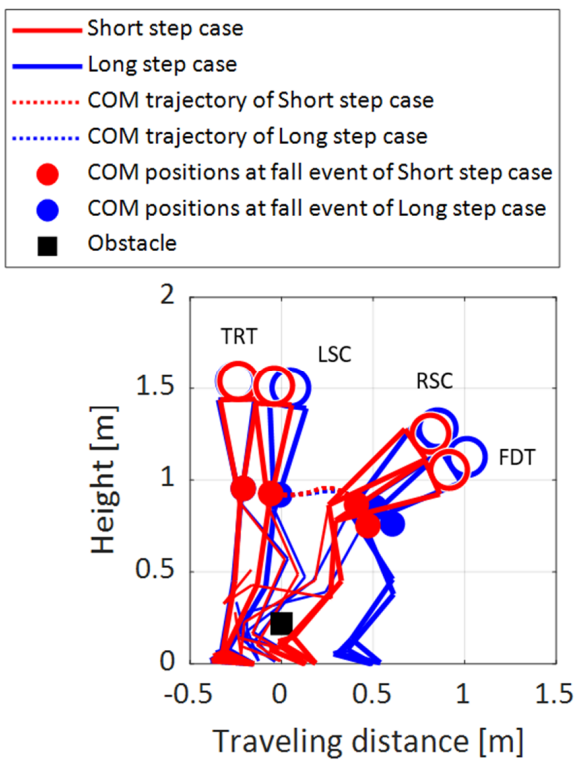

Fig. 7 Typical falling motion trajectory with different recovery step length Thick line shows recovery leg, thin line shows lowering leg

body speed became difficult in the short-step case at the RSC (Fig. 6). This result revealed that the CoM-CoP distance affected not only the balancing performance of the upstanding posture and normal gait (Hof et al., 2005; Hof, 2007; Winter, 1995), but also the mitigation motion against fall.

The ground impact force associated with a fall is an essential factor that determines the severity of injuries. However, it is impossible to actually measure such force because of ethical reasons. Thus, a model, which estimated the impact force from effective mass and speed at impact with the floor, was developed (DeGoede et al., 2003b). The effective mass, which affects the load applied to the impact area, is sometimes estimated as a part of body mass, especially when the body is supported by multiple contact points. Even in this study, the recovery leg supported most of body mass. However, the contribution of recovery leg probably decreased as CoM separated from the leg. Thus, the increase of CoM-CoP distance corresponded to the increase of the effective mass. In contrast, the vertical velocity of CoM and angular velocity of the trunk represents the fall speed. Although the large degree of freedom of body motion at the terminal phase of fall made it difficult to estimate the part and speed of impact, increase of these parameters suggested the increase of injury risk. Thus, our result suggested that the short-step case, whose CoM-CoP distance and CoM descending speed increased, would probably result in severe contact, in addition to less likelihood of avoiding a fall. Our result would also help in estimating the effective mass by extrapolating the observed fall motion.

\section{Conclusion}

A fall experiment, in which the subject would experience an actual fall, if not for a safety harness, was conducted 
to further understand the function of a recovery step against tripping during gait. Hence, the actual fall motion in the advanced phase, which was closer to the critical phase, was recorded and analyzed. The comparison of the physical parameters between different recovery step lengths identified the effect of it on the fall progression. Although the motion caused by the forward angular momentum caused by tripping was commonly observed, forward rotation and body descent could not be mitigated, especially in the short-step case. Furthermore, excessive forward sway of the CoM from the supporting point in the short-step case made it difficult to support the body because of the decrease of the GRF in the normal direction. This finding is important in estimating fall injuries or developing a mitigating method.

\section{Acknowledgments}

We thank the support by JSPS KAKENHI Grant Number 26750121 and the support of our group members, Ikuma Higo, Ryota Kushida, and Beltran Jessica. This work was supported by METI "Strategic international standardization promotion project: International standardization of human tolerance against fall injuries".

\section{References}

Arfken, C. L., Lach, H. W., Birge, S. J., and Miller, J. P., The prevalence and correlates of fear of falling in elderly persons living in the community, American Journal of Public Health, Vol.84, No.4 (1994), pp. 565-570.

De Leva P., Adjustments to Zatsiorsky-Seluyanov's segment inertia parameters, Journal of Biomechanics, Vol.29, No.9 (1996), pp. 1223-1230.

DeGoede, K. M., and Ashton-Miller, J., Fall arrest strategy affects peak hand impact force in a forward fall, Journal of Biomechanics, Vol.35, No.6 (2002), pp. 843-848.

DeGoede, K. M., and Ashton-Miller, J., Biomechanical simulations of forward fall arrests: effects of upper extremity arrest strategy, gender and aging-related declines in muscle strength, Journal of Biomechanics, Vol.36, No.3 (2003), pp. 413-420.

DeGoede, K. M., Ashton-Miller, J., and Schultz, A., Fall-related upper body injuries in the older adult: a review of the biomechanical issues, Journal of Biomechanics, Vol.36, No.7 (2003), pp. 1043-1053.

Dietz ,V., Quintern, J., Boos, G., and Berger, W., Obstruction of the swing phase during gait: phase-dependent bilateral leg muscle coordination, Brain Research, Vol.384, No.1 (1986), pp. 166-169.

Eng, J. J., Winter, D. A., and Patla, A. E., Strategies for recovery from a trip in early and late swing during human walking, Experimental Brain Research, Vol.102, No.2 (1994), pp. 339-349.

Grabiner, M. D., Koh, T. J., Lundin, T. M., and Jahnigen, D. W., Kinematics of recovery from a stumble, Journal of Gerontology, Vol.48, No.3 (1993), pp. M97-M102.

Hof, A., Gazendam, M., and Sinke, W., The condition for dynamic stability, Journal of Biomechanics, Vol.38, No.1 (2005), pp. 1-8.

Hof, A. L., The equations of motion for a standing human reveal three mechanisms for balance, Journal of Biomechanics, Vol.40, No.2 (2007), pp. 451-457.

Lipscomb, H. J., Glazner, J. E., Bondy, J., Guarini, K., and Lezotte, D., Injuries from slips and trips in construction, Applied Ergonomics, Vol.37, No.3 (2006), pp. 267-274.

Lo, J., and Ashton-Miller, J., Effect of pre-impact movement strategies on the impact forces resulting from a lateral fall, Journal of Biomechanics, Vol.41, No.9 (2008), pp. 1969-1977.

Luukinen, H., Herala, M., Koski, K., Honkanen, R., Laippala, P., and Kivelä, S. L., Fracture risk associated with a fall according to type of fall among the elderly, Osteoporosis International, Vol.11, No.7 (2000), pp. 631-634.

Mitsuoka, K., Akiyama, Y., Yamada, Y., and Okamoto, S., Analysis of Skip Motion as a Recovery Strategy after an Induced Trip, In: 2015 IEEE International Conference on Systems, Man, and Cybernetics, (2015). pp. 911-916.

Niino, N., Tsuzuku, S., Ando, F., and Shimokata, H., Frequencies and circumstances of falls in the National Institute for Longevity Sciences, Longitudinal Study of Aging (NILS-LSA), Journal of Epidemiology, Vol.10, No.1sup (2000) pp. 90-94.

Öberg, T., Karsznia, A., and Öberg, K., Basic gait parameters: reference data for normal subjects, 10-79 years of age, Journal of Rehabilitation Research and Development, Vol.30 (1993), pp. 210-210.

Owings, T. M., Pavol, M. J., and Grabiner, M. D., Mechanisms of failed recovery following postural perturbations on a 
motorized treadmill mimic those associated with an actual forward trip, Clinical Biomechanics, Vol.16, No.9 (2001), pp. 813-819.

Pavol, M. J., Owings, T. M., Foley, K. T., and Grabiner, M. D., Mechanisms leading to a fall from an induced trip in healthy older adults, The Journals of Gerontology Series A: Biological Sciences and Medical Sciences, Vol.56, No.7 (2001), pp. M428-M437.

Pijnappels, M., Bobbert, M. F., and van Dieën, J. H., Changes in walking pattern caused by the possibility of a tripping reaction, Gait \& Posture, Vol.14, No.1 (2001), pp.11-18.

Pijnappels, M., Bobbert, M. F., and van Dieën, J. H., Push-off reactions in recovery after tripping discriminate young subjects, older non-fallers and older fallers, Gait \& Posture, Vol.21, No.4 (2005), pp. 388-394.

Robinovitch, S. N., Feldman, F., Yang, Y., Schonnop, R., Leung, P. M., Sarraf, T., et al., Video capture of the circumstances of falls in elderly people residing in long-term care: an observational study, The Lancet, Vol. 381 (2013), pp. 47-54.

Schillings, A., Van Wezel, B., Mulder, T., and Duysens, J., Muscular responses and movement strategies during stumbling over obstacles, Journal of Neurophysiology, Vol.83, No.4, (2000), pp. 2093-2102.

Smeesters, C., Hayes, W. C., and McMahon, T. A., The threshold trip duration for which recovery is no longer possible is associated with strength and reaction time, Journal of Biomechanics, Vol.34, No.5 (2001), pp. 589-595.

Smeesters, C., Hayes, W. C., and McMahon, T. A., Disturbance type and gait speed affect fall direction and impact location, Journal of Biomechanics, Vol.34, No.3 (2001), pp. 309-317.

Tate, D. G., Workers' disability and return to work, American Journal of Physical Medicine \& Rehabilitation, Vol.71, No.2 (1992), pp. 92-96.

Wang, T. Y., Bhatt, T., Yang, F., and Pai, Y. C., Adaptive control reduces trip-induced forward gait instability among young adults, Journal of Biomechanics, Vol.45, No.7 (2012), pp. 1169-1175.

Winter, D. A., Human balance and posture control during standing and walking, Gait \& Posture, Vol.3, No.4 (1995), pp. 193-214.

Zhou, X., Draganich, L. F., and Amirouche, F., A dynamic model for simulating a trip and fall during gait, Medical Engineering \& Physics, Vol.24, No.2 (2002), pp.121-127. 